\title{
The 'stem cell' concept: is it holding us back?
}

\author{
Arthur D Lander
}

Address: Center for Complex Biological Systems, Department of Developmental and Cell Biology, and Department of Biomedical Engineering, University of California at Irvine, Irvine, CA 92697-2300, USA. Email: adlander@uci.edu

\begin{abstract}
Developmental biology, regenerative medicine and cancer biology are increasingly occupied with the molecular characterization of stem cells. Yet recent work adds to a growing body of literature suggesting that 'stemness' cannot be reduced to the molecular features of cell types, and is instead an emergent property of cell lineages under feedback control.
\end{abstract}

Great concepts in science do not just happen. They evolve, often along predictable lines. In an especially common scenario, an orderly set of related observations leads scientists to postulate an abstract entity or class whose existence somehow explains those relationships. Later on, the entity gets an unambiguous operational definition, through the application of some generally accepted experimental test. Frequently, in a third and final stage, the experimental test is replaced by a set of concrete features, typically physical, that more efficiently (if sometimes a little imperfectly) characterize the concept.

As an illustration of this progression, consider the concept 'gene'. The idea that inherited traits might be passed from parent to offspring through discrete units first led to abstract notions of some sort of 'unit of heredity'. Later, tests based on breeding and recombination allowed such a unit to be operationally defined in a rigorous (if sometimes laborious) way. Eventually, alternative definitions based on the physical characteristics of genes began to supplant such tests, so that most modern biologists now see genes as stretches of DNA with particular sequence characteristics, which happen to control inheritance (as opposed to units of heredity that happen to be instantiated in DNA). Evidence that this more physical definition of the gene has, for all practical purposes, replaced the one based on the operation of breeding can be seen in how readily we accept gene annotations for organisms, such as mammoths [1], which we could never actually breed.

Of course, not all scientific concepts get reduced to purely physical characteristics. But in the natural sciences, and especially biology, concepts that cannot be recast in this way often fail to develop much traction. One reason is that, to get a lot of use out of a concept, we need to be able to recognize quickly and easily what it refers to (we cannot afford to set up crosses every time we want to call something a gene). There is an even more compelling reason: history tells us that it is often only in the act of searching for the physical equivalents of abstract notions that we tend to learn whether those abstractions refer to anything real at all. As a case in point, consider 'phlogiston', an idea introduced in the seventeenth century to explain the process of combustion. The concept of phlogiston admits precise operational definition - it is the substance universally removed from all materials upon burning - but it happens that no substance with definite physical characteristics has ever been found that satisfies this operational definition. Indeed, it was ultimately the discovery that one of those physical characteristics would need to be the unlikely property of 'negative mass', which consigned phlogiston to the conceptual discard pile.

\section{Evolution of the stem cell concept}

Like gene or phlogiston, the term 'stem cell' is a scientific concept. Stem cells are very much in the news, thanks to a dramatic upsurge in interest in their therapeutic potential. The recent discovery that stem cell behaviors can be acquired by ordinary cells following the introduction of a small number of genes (reviewed in [2]) has intensified such interest. At the same time, the finding that only a small fraction of the cells within malignant tumors can initiate new tumors upon transplantation has led many cancer biologists to embrace the notion that stem cells are the driving force behind malignancies, and to advocate redirecting cancer therapy toward controlling or eradicating stem cells (reviewed in [3]). Clearly, we live in an era of biology when ideas and theories about stem cells are a major part of the intellectual landscape.

Where does stem cell as a concept lie along its evolutionary trajectory? The phrase seems to have come to us via histologists in the nineteenth century, who introduced it as a general, abstract term for cells specifically involved in repair or regeneration. With the discovery in the 1950 os that bone marrow cells could reconstitute the hematopoietic systems of irradiated individuals, the modern stem cell concept began to crystallize around the experimental procedures of transplantation and reconstitution. These and other studies gave us a good and enduring operational definition for stem cells: those cells that when introduced 
into a tissue depleted of its normal cells can, through proliferation and differentiation, reconstitute that tissue.

By this analysis, the stem cell concept finished passing through the stages of broad abstraction and precise operational definition by the 1960 s. Yet, despite no small amount of effort since then, a subsequent stage of evolution - in which stem cell is redefined in terms of physical characteristics - has yet to take place. It is perhaps curious that, after 45 years, we have been unable to place the general notion of 'stemness' on a purely molecular footing. Of course, the fact that a goal has not been achieved after a long time does not mean that the answer in not around the corner. But it does give one cause to wonder whether something we are doing needs to change, either the question we are asking or the way we are approaching it.

\section{Approaching stemness}

So far, the main way in which researchers have sought to get a handle on stemness has been to try to reduce stemcell behavior - a phenomenon operationally defined at the level of tissues and tissue reconstitution - to a set of necessary and sufficient, intrinsic cell-level properties. The two properties that are universally discussed are 'potency' and 'self-renewal'. Stem cells, it is said, display traits of potency and self-renewal that set them apart from other cells. Understanding the molecular basis of these abilities, it is argued, should lead directly to a molecular description of stemness. In evaluating this plan, we need ask two questions. First, do stem cells really perform feats of potency and self-renewal that set them apart? Second, should we expect there to be a common molecular basis for those behaviors?

Both of these questions are surprisingly thorny. Consider the matter of potency: the usual definition is the ability to generate cells of other types, but there are two problems with this. First, there are clearly cells in the body that generate cell types other than themselves but which are not regarded as stem cells (for example, those that are often called 'committed progenitors'). We can distinguish such cells from stem cells by requiring the latter to be at least bi-potent (able to produce at least two cell types other than themselves), but it is not clear that adult stem cells necessarily have this property. And if we intend to include cancer stem cells in our definition, we would be hard pressed to find any good reason to demand that those cells produce more than one type of differentiated offspring. A converse issue arises during embryonic development, because the vast majority of cells, at early stages at least, go through many sequential multipotent stages. Unless we wish to call all of them stem cells (which would seem to rob the concept of much of its utility), we are forced to conclude that high potency alone is an insufficiently restrictive criterion by which to define stem cells.
The second problem with defining stem cells in terms of potency is that traditional notions of what a cell type is, and how cells move between types, seem to be eroding, thanks to recent work by stem cell researchers and an influx of ideas from systems biology. The traditional view of cell differentiation as a set of irreversible, deterministic transitions from one stable state to another is giving way to a view in which cell states are quasi-stable points on an 'energy landscape' along which cells move in response to both stochastic variation and external signals (see, for example, [4-6]). By this view, potency is not really a property that a cell has independent of its environment. A good analogy likens cell types to metabolites in metabolic networks. Arrow diagrams showing pathways by which glucose can be converted into hundreds of other substances make nice wall charts, but if one wants to say what glucose will actually become in any real situation, one needs to know enzyme levels and activities, levels of other metabolites, temperature, $\mathrm{pH}$ and so forth.

From the above discussion, we might argue that a definition of stemness based solely on potency suffers from being either too restrictive, too inclusive or too unhelpful. Of course, this does not stop us from adopting a definition that combines potency with some other property, such as self-renewal. Indeed, this seems to be the position that most stem cell researchers currently adopt.

Unfortunately, self-renewal is an even more slippery notion than potency. To begin with, the most self-evident meaning of this phrase - making more of one's self - is clearly not what is meant when it is used in the stem cell field. Even terminally differentiated cells (as long as they are not post-mitotic) are self-renewing in this sense. Rather, what is generally meant by self-renewal is that stem cells maintain their own numbers at the same time as they are producing cells of other types. A more accurate, if cumbersome, phrase might be 'numerical homeostasis in the face of constant differentiation'.

On the face of it, such homeostasis is indeed a remarkable phenomenon, and worthy of investigation. But is it a necessary feature of stem cells? And when it occurs, can it be reduced to a set of unique molecular characteristics? Unfortunately, the odds that either question can be answered in the affirmative do not look good.

To begin with, the only stem cells for which we can expect to see homeostasis of cell number are those of mature tissues (so-called 'adult' stem cells). During embryonic development we should want stem cell populations to grow over time. Likewise, cancer stem cell populations, by the very definition of cancer, expand. So strict adherence to homeostasis would eliminate from consideration too many of the cells we would like to include as stem cells. Yet even if we restrict ourselves to adult stem cells, we still run into problems. To 
see why, it is helpful to think about the possible mechanisms by which homeostasis might be achieved.

The first such mechanism is for every stem cell to undergo obligatory asymmetric division. According to this model, when a stem cell divides, one daughter differentiates and one remains a stem cell. The alternative model, sometimes referred to as 'stochastic differentiation', has stem cells making a mixture of asymmetric divisions and symmetric ones, the latter producing either two stem cells (symmetric renewal), or two differentiated cells (symmetric differentiation). In this case, homeostasis is achieved if, and only if, the two types of symmetric divisions occur with exactly equal probabilities. The need for exact balance is underscored by the simple calculation that a mere $10 \%$ deviation from equality would, if sustained, cause the stem cells in a tissue to either expand indefinitely or vanish, with a doubling (or halving) time of about seven cell cycles.

Traditionally, the all-asymmetric-division model is seen as the simpler of the two, and it is certainly the more popular in textbooks. The suggested mechanism is that stem cells distribute different cytoplasmic 'determinants' to their apical and basal ends. By constraining cell division planes to lie perpendicular to the apico-basal axis, determinants of stemness necessarily pass to just one of the two daughter cells. The major difficulty with this model is that it works too well: if stem cell populations are ever to expand (for example, during embryonic development), there needs to be some way either to shut off the differential sorting of determinants, or reorient planes of cell division.

\section{The benefits of feedback}

Just as the difficulty with the all-asymmetric division model is getting it to do anything other than homeostasis, the perceived difficulty with the stochastic model has always been in getting it to achieve homeostasis at all. Specifically, how could a population of individual, stochastically-acting cells assure that their rates of renewal and differentiation are exactly balanced?

Interestingly, this problem has recently been solved, and the answer is simplicity itself: all that is required is for the descendants of stem cells to release a substance that accumulates according to their abundance, and feeds back to decrease the renewal probability of stem cells (that is, increase the fraction of stem cell progeny that differentiate). As the consequence of an engineering principle known as 'integral feedback control', an exact balance of renewal and differentiation automatically occurs, regardless of how the feedback substance works, how much of it is made or how sensitive cells are to it [7].

In fact, research showing that self-maintaining tissues do release feedback regulators (chalones) goes back to the 1950s, and received a big boost in the 1990 s with the discovery of myostatin and its role as a feedback controller of muscle growth [8]. Since then, several other chalones have been identified in various tissues, many of which, like myostatin, are members of the TGF $\beta$ family of growth factors. Although it was originally thought that chalones acted by regulating the rates at which cells divide, recent work in olfactory epithelium [7] and muscle [9] shows that just as is needed by the stochastic differentiation model they also decrease the probability that stem and progenitor cell progeny remain at the stem or progenitor cell stage (that is, they increase the probability of differentiation). Thus, the machinery for achieving stem cell-driven tissue maintenance in the face of stochastic stem cell behaviors is clearly implemented in at least some tissues.

The jury is still out on whether all self-maintaining tissues exploit stochastic renewal with feedback or whether some, do in fact rely on invariant asymmetric stem cell divisions. A strong prediction of the stochastic model is that even in the face of homeostasis, every stem cell pedigree has a nonzero probability of going extinct. By pedigree I refer here to a stem cell and its (non-stem cell) descendants, all of which would be doomed to disappear whenever their stem cell ancestor undergoes symmetric differentiation. In fact, periodic random extinction (balanced by an equal rate of pedigree duplication when stem cells undergo symmetric renewal) provides one of the most plausible explanations for the extremely high variability commonly seen in the sizes of stem cell-derived clones in vivo $[10,11]$. Other kinds of experimental data also support the occurrence of pedigree extinction, sometimes also referred to as 'niche succession' (see, for example, [12,13]). The main problem with such extinctions, in theory at least, is that they make it easy for pathologically 'advantaged' stem cells (for example, ones that have, through mutation, become faster -growing or less susceptible to feedback control) to displace normal ones. The preference for asymmetric division by stem cells that is observed in many tissues might well have arisen as a protective mechanism to minimize this effect. Put another way, the fact that stem cells very often do divide asymmetrically in no way implies that asymmetric division is the mechanism of tissue homeostasis. One can achieve homeostasis through feedback control, and still expect to see frequent (albeit not obligatory) asymmetric division, especially in tissues with rapid turnover in which stem cells must divide many times during the lifetime of the organism.

The control of stem cell behaviors through feedback regulation of self-renewal is impressive not only for the ease and flexibility with which it achieves homeostasis, but even more so for what it accomplishes under other circumstances: when tissues are away from equilibrium (that is, have more or fewer differentiated cells than the level at which homeostasis would be achieved), the alteration in feedback causes stem cell populations to either expand or 
contract exponentially - producing a remarkably rapid return toward equilibrium $[7,14]$. This phenomenon explains many dynamic features of tissue growth and regeneration. For example, during regeneration it is common to observe progenitor pools transiently expand and contract, the latter occurring in concert with the reappearance of differentiated cells. In developing tissues, expanding and contracting progenitor pools are also commonly observed, and in at least one case the self-renewal probabilities of progenitors have been measured and found to decline continuously as differentiated cells appear [15]. All these behaviors are predicted by feedback regulation models [7,14]. Such models also show how oscillations easily emerge in the production of differentiated cells (such as are observed in the hair cycle, and in certain hematopoietic and immune conditions) [14], and explain why stem cell numbers selfregulate (for example, why one can introduce large numbers of additional embryonic stem cells into a mouse blastocyst yet end up with a normal mouse). Finally, feedback control provides a general explanation for why stem cells seem to behave homeostatically only in their normal in vivo context (an idea, which suggests that a stem cell's 'niche' may not just be an environment in which it thrives, but also one in which it can exercise its own potential for self-control).

\section{Do principles of control define a unique class of cells?}

The fact that so many characteristic behaviors of stem cells and stem cell systems - homeostasis in the face of the production of differentiated progeny; rapid tissue regeneration; developmental expansion; self-regulation; and niche dependence - emerge simply as a result of feedback regulation of self-renewal suggests that the ability to be regulated by such feedback might serve as a good defining characteristic of stemness. Alas, we have no such luck here.

As it happens, cells that are not considered stem cells also show this behavior, namely the so-called 'transit amplifying cells' that lie downstream of stem cells in tissue lineages, and are distinguished by an apparently limited potential for self-renewal. For example, in the olfactory epithelium, qualitatively similar feedback effects are mediated by two different TGF $\beta$ family members upon the stem cell and its molecularly distinct descendant, respectively [7]. That descendant, the immediate neuronal progenitor, behaves like a typical committed progenitor, generally selfrenewing only a few times before differentiating [16].

In fact, it is probably no accident that, even within a single lineage, both stem and transit amplifying cells are subject to the same sort of feedback regulation. To see why, consider the diagram in Figure 1, which depicts a threestage lineage progressing from what might be a stem cell (type o) to a transit amplifying cell (type 1), to a postmitotic differentiated cell (type 2). Each dividing cell type

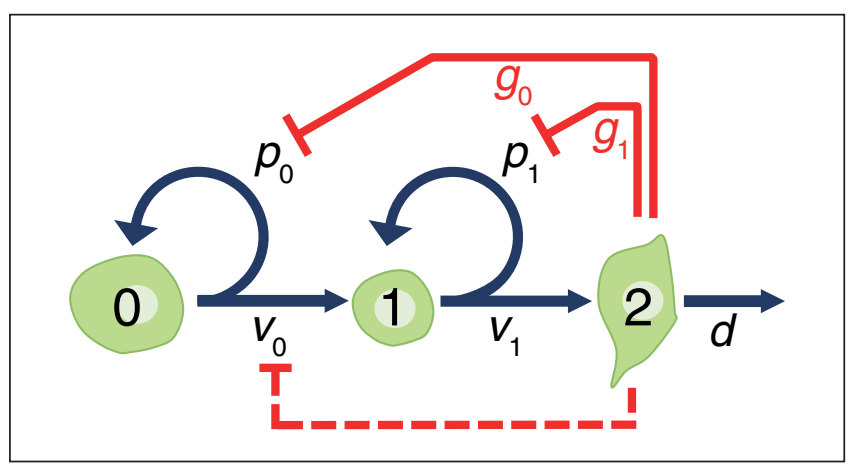

Figure 1

Feedback produces stem cell and transit amplifying cell behaviors. A two-stage cell lineage is shown, in which cells of type 0 and type 1 divide (at rates $v_{0}$ and $v_{1}$, respectively), and produce progeny with an intrinsic probability ( $p_{0}$ or $p_{1}$, respectively) of remaining at the same lineage stage (as opposed to differentiating to the next one).

Cell type 2 is terminally differentiated, and dies (or is shed) at rate $d$. As long as $p_{0}>0.5$, any negative feedback (schematized by solid red lines, and quantified by parameters $g_{1}$ and $g_{2}$ ) from cell type 2 onto $p_{0}$ forces the system to reach a self-maintaining steady state. If there is feedback onto both $p_{0}$ and $p_{1}$, the quantitative details determine whether cell type 0 behaves like a stem cell and cell type 1 like a transit amplifying cell; or whether cell type 1 behaves like a stem cell and cell type 0 goes extinct. If feedback also slows cell division (for example, $v_{0}$, dashed red line), more complex system behaviors may occur (for example, in which cell type 1 normally fulfills most stem cell functions, with cell type 0 remaining quiescent except following tissue injury, when it transiently 'reawakens'). For further details see $[7,14,17]$.

is characterized by an intrinsic probability of renewal, $p$, which quantifies that overall fraction of its progeny that remains at the same lineage stage, and by a feedback gain $g$, which quantifies how the cell's actual renewal probability declines with the number of terminally differentiated cells in its vicinity. Consider now the case when both $p_{0}$ and $p_{1}$ are greater than 0.5 ; that is, the intrinsic tendency of both type 0 and type 1 cells is to renew more than half the time, and it is only feedback that restrains them from doing so. Depending on the values of the $p$ - and $g$-parameters, this system will behave in one of two ways.

In one parameter regime - when the amount of feedback needed to lower the renewal behavior of cell type o to $50 \%$ is sufficient to drive that of cell type 1 below 50\% - the system will achieve a stable steady state in which cell type o maintains its numbers homeostatically and cell type 1 displays frequent random extinctions. Unless one is looking closely at the statistics of such events, the behavior of cell type 1 will suggest that it has only a limited, relatively fixed potential to self-renew.

In the other parameter regime - in which the amount of feedback needed to lower the renewal behavior of cell type o to $50 \%$ is not sufficient to drive that of cell type 1 to $50 \%$ - 
something completely different will happen. Cell type o will be driven to extinction, and cell type 1 will be driven to homeostasis.

In other words, which cell becomes the stem cell in this simple system is just a matter of parameter values, nothing more $[7,14,17]$. And since these values depend on things like the numbers of differentiated cells and the amounts of the various feedback factors they produce, we would have a hard time avoiding the conclusion that it is cellular context, not intrinsic molecular specification, that establishes whether a cell type becomes a stem cell, a transit amplifying cell or simply goes extinct.

\section{Beyond stemness?}

The view that stem cells are not fixed in their proliferative behaviors reinforces suggestions by others - mostly motivated by the problems that arise when trying to define stem cells in terms of potency - that the term stem cell properly applies only to 'condition', not 'character' $[18,19]$. If this is really the case, what does it say about the many attempts over the years to define stemness in terms of molecular properties?

Above all, it suggests that stemness is a property of systems, rather than cells, with the relevant system being, at minimum, a cell lineage, and more likely a lineage plus an environment. A system with stemness is typically one that can achieve a controlled size, maintain itself homeostatically, and regenerate when necessary. Moreover, it most probably does so by exploiting basic principles of feedback control.

If stemness is a system-level property, then the concept of stem cell is really fundamentally different from that of, say, gene. A more similar concept might be something like 'rate-limiting enzyme', which also defines a class of tangible, physical objects, but only does so in terms of their functional roles within a system, not their intrinsic biochemical properties.

The assertion that the stem cell concept cannot be reduced to the molecular properties of individual cells is more than just an esoteric philosophical stance; it has important practical ramifications. For one thing, it suggests that the kind of molecular understanding that researchers most urgently need to pursue is of basic cellular phenomena that, while not unique to stem cells, are critical for stem cell function: for example, the ability of daughter cells to take on fates different from their mothers; the ability of sister cells to take on fates different from each other; and the ability of external cues to regulate both of these properties.

For another, the observation that stem cell behaviors can emerge as a consequence of feedback control calls attention to the fact that stem cell systems are, fundamentally, dynamical systems. Their behaviors can be complex and counterintuitive, yet ultimately still understandable, especially with the help of modeling or simulation. Given that lineage relationships and feedback configurations can be far more elaborate than those shown in Figure 1, concerted efforts are needed to elucidate the classes of dynamical behaviors of which stem cell systems are capable. For example, in the case of cancers that are stem cell driven, it is not clear that we actually have grounds to assume that the specific chemotherapeutic targeting of cancer stem cells will necessarily stop tumors in their tracks. Indeed, if feedback and lineage progression continue to take place in cancerous tissues, we might observe that, under different conditions - different stages of tumorigensis, different parts of a tumor, different amounts of tumor cells - different cell types will assume the role of 'cancer stem cells'. The therapeutic implications of this possibility are clearly substantial.

In summary, it would seem that the concept of stem cell indeed has the potential to hold us back - especially if we focus on demanding from it things it cannot give. But if we can re-fashion our thinking at a different level - in which systems relationships and dynamics take the place of molecular signatures and simple gene regulatory circuits then there is a chance that the concept of stem cell will continue to light the path toward biological understanding.

\section{Acknowledgements}

I thank Anne Calof, John Lowengrub, Qing Nie and Fred Wan for helpful discussions. Some of the themes discussed in this piece have also been articulated by others, and I regret that, because of space constraints, it was not possible to cite all of the relevant literature.

\section{References}

1. Miller W, Drautz DI, Ratan A, Pusey B, Qi J, Lesk AM, Tomsho LP, Packard MD, Zhao F, Sher A, Tikhonov A, Raney B, Patterson N, Lindblad-Toh K, Lander ES, Knight JR, Irzyk GP, Fredrikson KM, Harkins TT, Sheridan S, Pringle T, Schuster SC: Sequencing the nuclear genome of the extinct woolly mammoth. Nature 2008, 456:387-390.

2. Jaenisch R, Young R: Stem cells, the molecular circuitry of pluripotency and nuclear reprogramming. Cell 2008, 132: 567-582.

3. Marotta LL, Polyak K: Cancer stem cells: a model in the making. Curr Opin Genet Dev 2009, 19:44-50.

4. Enver T, Pera M, Peterson C, Andrews PW: Stem cell states, fates, and the rules of attraction. Cell Stem Cell 2009, 4:387397.

5. Chang $\mathrm{HH}$, Hemberg $\mathrm{M}$, Barahona $\mathrm{M}$, Ingber DE, Huang S: Transcriptome-wide noise controls lineage choice in mammalian progenitor cells. Nature 2008, 453:544-547.

6. Chuong CM, Widelitz RB: The river of stem cells. Cell Stem Cell 2009, 4:100-102.

7. Lander AD, Gokoffski KK, Wan FY, Nie Q, Calof AL: Cell lineages and the logic of proliferative control. PLOS Biol 2009, 7:e15.

8. Lee SJ, McPherron AC: Myostatin and the control of skeletal muscle mass. Curr Opin Genet Dev 1999, 9:604-607.

9. Manceau M, Gros J, Savage K, Thome V, McPherron A, Paterson $B$, Marcelle C: Myostatin promotes the terminal differentia- 
tion of embryonic muscle progenitors. Genes Dev 2008, 22: 668-681.

10. Till JE, McCulloch EA, Siminovitch L: A stochastic model of stem cell proliferation, based on the growth of spleen colony-forming cells. Proc Natl Acad Sci USA 1964, 51:29-36.

11. Mangel $M$, Bonsall $M B$ : Phenotypic evolutionary models in stem cell biology: replacement, quiescence, and variability. PLOS ONE 2008, 3:e1591.

12. Jordan CT, Lemischka IR: Clonal and systemic analysis of long-term hematopoiesis in the mouse. Genes Dev 1990, 4: 220-232.

13. Kim KM, Shibata D: Methylation reveals a niche: stem cell succession in human colon crypts. Oncogene 2002, 21: 5441-5449.

14. Lo WC, Chou CS, Gokoffski KK, Wan FY, Lander AD, Calof AL, Nie Q: Feedback regulation in multistage cell lineages. Math Biosci Eng 2009, 6:59-82.

15. Takahashi T, Nowakowski RS, Caviness VS Jr: The mathematics of neocortical neuronogenesis. Dev Neurosci 1997, 19: $17-22$.
16. Wu HH, Ivkovic S, Murray RC, Jaramillo S, Lyons KM, Johnson $\mathrm{JE}$, Calof AL: Autoregulation of neurogenesis by GDF11. Neuron 2003, 37:197-207

17 Johnston MD, Edwards CM, Bodmer WF, Maini PK, Chapman SJ: Examples of mathematical modeling: tales from the crypt. Cell Cycle 2007, 6:2106-2112.

18. Zipori $D$ : The nature of stem cells: state rather than entity. Nat Rev Genet 2004, 5:873-878.

19. Loeffler M, Roeder I: Tissue stem cells: definition, plasticity, heterogeneity, self-organization and models - a conceptual approach. Cells Tissues Organs 2002, 171:8-26.

See other regeneration and stem cells articles $h$ ttp://jbiol.com/content/9/2/14 and http://jbiol.com/content/9/2/15

Published: 21 September 2009

doi:10.1186/jbiol177

(C) 2009 BioMed Central Ltd 